\title{
Review of: "A broad-spectrum and highly potent human monoclonal antibody cocktail for rabies prophylaxis"
}

\author{
Omesh Kumar Bharti
}

Potential competing interests: The author(s) declared that no potential competing interests exist.

Recently in 2018, WHO has recommended use of monoclonal antibody (mAb) "cocktails" containing at least two antibodies against RABV, as alternatives for Rabies Immunoglobulins (RIG) in Rabies Post Exposure Prophylaxis (PEP). Several human mAbs have been tested against rabies. Studies so far show the equivalence of its performance to human RIG (HRIG). The availability of this mAb could fill critical public health gaps. As it is made by recombinant technology, it will be less prone to problems such as availability, safety and purity. It should be recommended for use in public health programmes, depending on the epidemiological and geographical setting, with monitoring of its safety and efficacy (clinical outcomes) during post-marketing use (WHO TRS 1012).

The authors need all the appreciation for developing a noble mABs cocktail that supplements effect of each other than interfering and effectively neutralizes broad range of lyssaviruses of clinical importance. Phage display technology use by the authors is a new handle to develop new drugs and that has been used by the authors brilliantly in developing this mAbs cocktail.

A mouse challenge study was performed by the authors using rabies virus isolated from infected dogs and humans in India. After 3 hours of virus inoculation each mAb was inoculated at same site at a dose of 20 $\mathrm{IU} / \mathrm{Kg}$, confuses the reader a little as, while advocating only wound infiltration of mAbs as per reference no. 13 in the paper (Bharti et al) the required mAb at the site of virus inoculation may be of less volume as per the studies done recently by Bharti et al and Madhusudana et al.

(https://pubmed.ncbi.nlm.nih.gov/31395454/) (https://pubmed.ncbi.nlm.nih.gov/23792347/)

WHO in its latest recommendations advocates IM administration of RIG only in cases where wounds are invisible say exposure to Bats. So it is advised that mAbs volumes in this study for local wound infiltration be analysed separately (as detailed in the above reference by Madhusudana et al) and the current calculated volume of this mAbs at $20 \mathrm{IU} / \mathrm{Kg}$ may be kept for rare opportunities where wounds are not visible and IM administration of this mAbs cocktail is required. This shall help in wider use by the patients and make this product affordable to the poor.

Though it has been explained in the study that all type of Indian rabies viruses were evaluated in the experiment in vivo and in vitro (Fig 3) still it could have been better to evaluate specifically the viruses of Indian Mongoose and Jackle, that are clinically relevant in India, to give more confidence to clinicians in India. 
This study is a hope for the next generation mAbs against rabies and it is hopeful that further human studies would prove the efficacy of this cocktail and soon the victims shall get the benefit of this new cocktail world-over. 